\title{
Influence of Ionic Strength on the Deposition of
}

\section{Metal-Phenolic Networks}

Junling Guo, ${ }^{+,+}, \perp$ Joseph J. Richardson, ${ }^{+, \xi, \perp}$ Quinn A. Besford, ${ }^{\dagger, t}$ Andrew J. Christofferson,

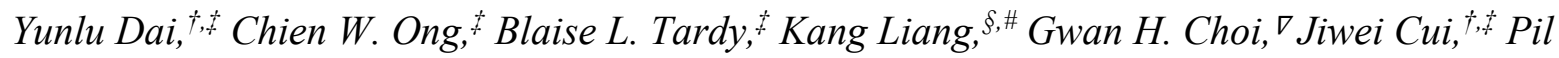
J. Yoo, ${ }^{\nabla}$ Irene Yarovsky, "and Frank Caruso ${ }^{*},+$,

${ }^{\dagger}$ ARC Centre of Excellence in Convergent Bio-Nano Science and Technology, The University of Melbourne, Parkville, Victoria 3010, Australia

Department of Chemical Engineering, The University of Melbourne, Parkville, Victoria 3010, Australia

${ }^{\S}$ CSIRO Manufacturing Flagship, CSIRO, Clayton South, Victoria 3169, Australia

"School of Engineering, RMIT University, GPO Box 2476, Melbourne, Victoria 3001, Australia.

${ }^{\#}$ School of Chemical Engineering and Graduate School of Biomedical Engineering, The University of New South Wales, Sydney, NSW 2052, Australia

${ }^{\nabla}$ School of Chemical Engineering and SKKU Advanced Institute of Nanotechnology (SAINT), Sungkyunkwan University (SKKU), Suwon 16419, Republic of Korea

${ }^{\perp}$ These authors contributed equally to this work

*Corresponding author. E-mail: fcaruso@unimelb.edu.au 
Metal-phenolic networks (MPNs) are a versatile class of self-assembled materials that are able to form functional thin films on various substrates with potential applications in areas including drug delivery and catalysis. Different metals (e.g., iron, copper) and phenols (e.g., tannic acid, gallic acid) have been investigated for MPN film assembly; however, a mechanistic understanding of the thermodynamics governing MPN formation remains largely unexplored. Thus far, MPNs have been deposited at low ionic strengths $(<5 \mathrm{mM})$, resulting in films with typical thicknesses of $\sim 10$ $\mathrm{nm}$, and it is still unclear how a bulk complexation reaction results in homogenous thin films when a substrate is present. Herein, we explore the influence of ionic strength $(0-2 \mathrm{M} \mathrm{NaCl})$ on the conformation of MPN precursors in solution and how this determines the final thickness and morphology of MPN films. Specifically, the film thickness increases from $10 \mathrm{~nm}$ in $0 \mathrm{M} \mathrm{NaCl}$ to $12 \mathrm{~nm}$ in $0.5 \mathrm{M} \mathrm{NaCl}$, and to $15 \mathrm{~nm}$ in $1 \mathrm{M} \mathrm{NaCl}$, after which the films grow rougher rather than thicker. For example, the root mean square roughness values of the films are constant below $1 \mathrm{M}$ $\mathrm{NaCl}$ at $1.5 \mathrm{~nm}$; in contrast, at $1 \mathrm{M} \mathrm{NaCl}$, the roughness is $3 \mathrm{~nm}$ and at $2 \mathrm{M} \mathrm{NaCl}$, the roughness value increases to $5 \mathrm{~nm}$. Small-angle X-ray scattering and molecular dynamics simulations allow for comparisons to be made with chelated metals and polyelectrolyte thin films. For example, at a higher ionic strength $(2 \mathrm{M} \mathrm{NaCl})$, sodium ions shield the galloyl groups of tannic acid, allowing them to extend away from the $\mathrm{Fe}^{\mathrm{III}}$ center and interact with other MPN complexes in solution to form thicker and rougher films. As the properties of films determine their final performance and application, the ability to tune both thickness and roughness using salts may allow for new applications of MPNs. 


\section{INTRODUCTION}

Metal-phenolic networks (MPNs) composed of polyphenols and metal ions allow for the versatile engineering of functional surfaces and particles. The natural adherent properties of polyphenols enable MPN film deposition on various substrates, ${ }^{1}$ where the functionality of the films can be controlled through choice of the metal ions and ligands. ${ }^{2,3}$ Moreover, various phenolic building blocks have been used for MPN film formation (e.g., tannic acid, gallic acid, pyrocatechol), ${ }^{4}$ and the resultant films have been well characterized in terms of material properties (e.g., thickness, permeability, stiffness). ${ }^{1,4,5}$ However, the mechanisms behind MPN film formation remain largely unexplored. Investigating the influence of the thermodynamic parameters governing MPN deposition is important to increase our understanding of MPN films and the deposition process.

The two primary thermodynamic factors surrounding chelation complexes are temperature and ionic strength, ${ }^{6-8}$ with neither previously studied for MPN-assembled films. The effects of ionic strength have been determined for non-MPN chelation complexes in solution, ${ }^{8}$ and temperature generally plays a smaller role in changing stability constants owing to the relatively small size of chelation complexes. ${ }^{6,7,9,10}$ Most studies have been performed with single-metal chelators (e.g., ethylenediaminetetraacetic acid, egtazic acid), where increased salt concentrations decrease the association constants of the chelated metals, (i.e. salts destabilize the complex). However, MPNs are often composed of multidentate polyphenols capable of interacting with numerous metal ions at numerous interaction sites. Therefore previous literature investigations into non-MPN single metal ions complexed with single-metal chelators may not fully explain the interactions governing the formation of MPN films. Contrarily, polyelectrolyte complexes have multiple interaction sites and have been studied at different ionic strengths both in solution and films. ${ }^{11-17}$ For example, at 
low ionic strengths, charge repulsion leads to the formation of rod-like, extended polymers in the bulk solution, potentially resulting in reduced coverage and thinner films when deposited on a substrate. ${ }^{1-17}$ Conversely, at higher ionic strengths, charge repulsion is shielded and polymers have a globular morphology in the bulk, leading to the deposition of rougher, inhomogeneous films on a substrate. ${ }^{11,12}$ Because polyelectrolyte complexes and polymers in solution are governed by charge-charge interactions, a complete comparison with MPNs is challenging, as MPNs have characteristics of both chelated metals and polyelectrolytes. Investigating how ionic strength differences influence MPNs will help elucidate the thermodynamics governing MPN film deposition and provide insights into the parallels between chelated metals, polyelectrolytes, and MPNs. This information would allow for improved engineering of MPN films and has implications for applications such as electronics, drug delivery, biocatalysis, and coatings.

Herein, we report and characterize the effects of ionic strength on MPN film deposition and the formation of complexes in solution using iron-tannic acid (Fe $\left.\mathrm{Fe}^{\mathrm{III}}-\mathrm{TA}\right)$ complexes (Figure 1). ${ }^{1}$ As seen with polyelectrolyte films, high ionic strengths lead to thicker and rougher films. Furthermore, similarly to chelated metals, high ionic strengths alter the MPN complex structure, allowing some galloyl groups on TA to extend out from the Fe $\mathrm{FII}^{\mathrm{III}}$ center and interact with other complexes. The change from homogeneous thinner films to rougher and thicker films suggests a cross-over in the mechanism for the deposition of MPNs. Molecular dynamics (MD) simulations confirm these findings and provide insights into the structure of the $\mathrm{Fe}^{\mathrm{III}}-\mathrm{TA}$ complexes prior to film deposition. We find that the presence of positively charged counterions (e.g., sodium ions) is essential for enabling phenolic moieties to extend into solution. These results allow for an additional degree of control over MPN film engineering and provide a better understanding of the molecular behavior of $\mathrm{Fe}^{\mathrm{III}}$-TA complexes during the film assembly process. 


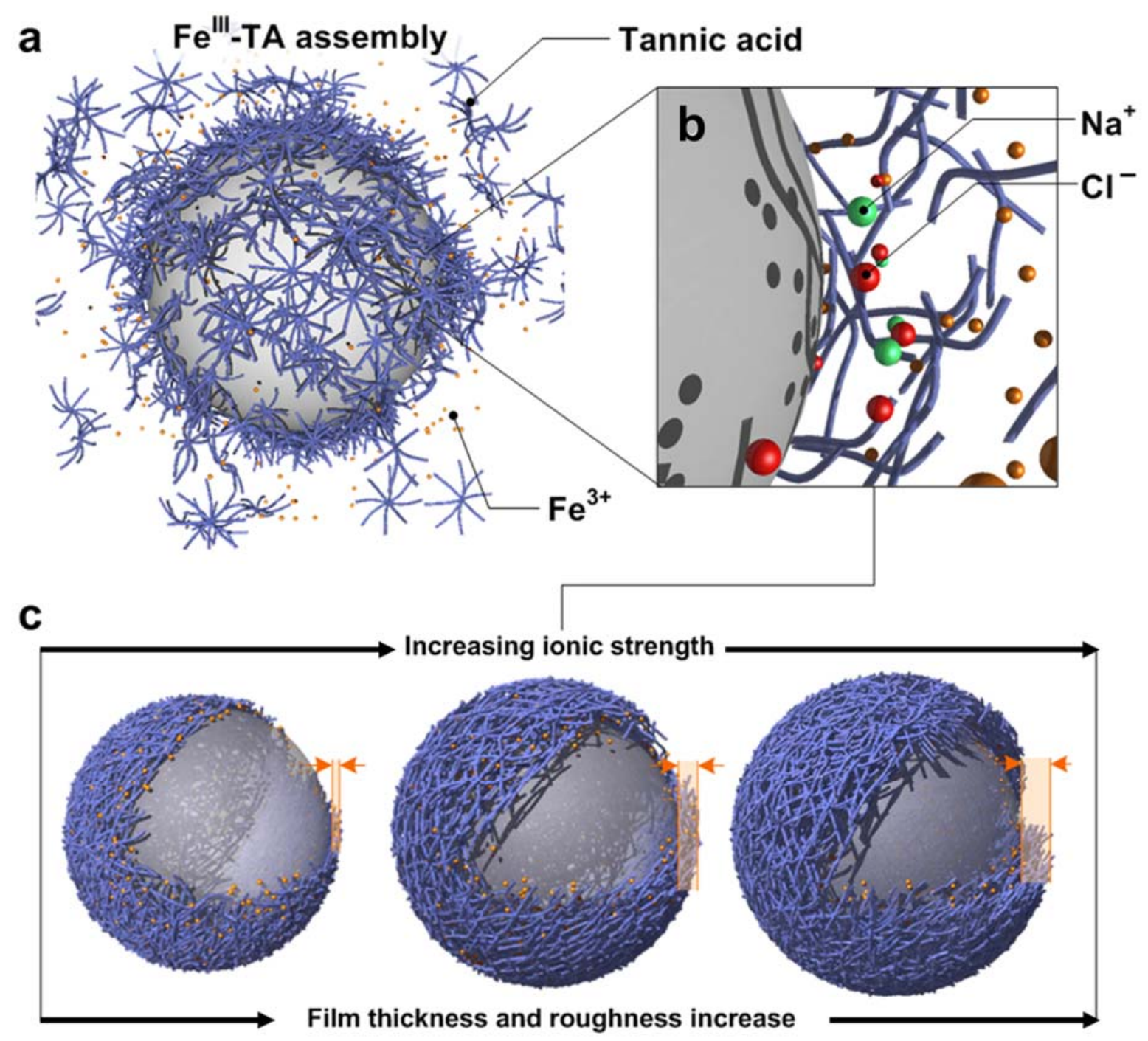

Figure 1. Schematic illustrations of (a) MPN film assembly in the presence of $\mathrm{NaCl}$ and (b) magnified area highlighting the presence of counterions. (c) Thicker and rougher MPN films are produced at higher salt concentrations. 
Materials. Tannic acid (TA), iron(III) chloride hexahydrate $\left(\mathrm{FeCl}_{3} \cdot 6 \mathrm{H}_{2} \mathrm{O}\right)$, tris(hydroxymethyl)aminomethane (Tris), (3-(N-morpholino)propanesulfonic acid) (MOPS), and sodium chloride $(\mathrm{NaCl})$ were purchased from Sigma-Aldrich. Tetrahydrofuran (THF) was purchased from Chem-Supply. All chemicals were used as received. High-purity Milli-Q water with a resistivity of $18.2 \mathrm{M} \Omega . \mathrm{cm}$ was obtained from an inline Millipore RiOs/Origin water purification system.

\section{Deposition of Fe $\mathrm{FII}^{\mathrm{II}} \mathrm{TA}$ Films on Particle Templates with Different Salt Concentrations.}

Fe $\mathrm{III}^{\mathrm{III}} \mathrm{TA}$ film formation was performed on selectively degradable particle templates. After film formation, the templates were removed with an organic solvent to produce hollow free-standing Fe ${ }^{\text {III }}$-TA films (capsules).

The experimental protocol was based on our previously reported film formation method. ${ }^{1}$ Briefly, $50 \mu \mathrm{L}$ polystyrene (PS) particles (diameter $=3.55 \mu \mathrm{m}$ ) was first added to $440 \mu \mathrm{L}$ aqueous $\mathrm{NaCl}$ solution. Next, $5 \mu \mathrm{L} \mathrm{FeCl}_{3} \cdot 6 \mathrm{H}_{2} \mathrm{O}\left(10 \mathrm{mg} \mathrm{mL}^{-1}\right)$ and subsequently $\mathrm{TA}\left(40 \mathrm{mg} \mathrm{mL}^{-1}\right)$ solutions were added to the $490 \mu \mathrm{L}$ PS template suspension to yield final $\mathrm{FeCl}_{3} \cdot 6 \mathrm{H}_{2} \mathrm{O}$ and TA concentrations of 0.1 and $0.4 \mathrm{mg} \mathrm{mL}^{-1}$, respectively. The final concentration of $\mathrm{NaCl}$ was altered from the standard conditions $(0 \mathrm{M})^{1}$ to higher salt concentrations $(0.2,0.5,0.75,1.0,1.5$, and $2.0 \mathrm{M})$. The suspension was vigorously mixed by a vortex mixer for $10 \mathrm{~s}$ immediately after the individual additions of $\mathrm{FeCl}_{3} \cdot 6 \mathrm{H}_{2} \mathrm{O}$ and TA. The $\mathrm{pH}$ of this suspension was then raised by adding $0.5 \mathrm{~mL}$ MOPS (20 mM, pH 7.4). The particles were washed with water thrice to remove excess TA and $\mathrm{FeCl}_{3}$. In the washing step, the particles were spun down by centrifugation $(2000 \mathrm{~g}, 30 \mathrm{~s})$ and the supernatant was removed. To obtain the Fe $\mathrm{E}^{\mathrm{III}}-\mathrm{TA}$ capsules, the PS was removed by washing with 
THF four times. In each THF washing step, the pellets were first vortexed for $10 \mathrm{~s}$. Then, $700 \mu \mathrm{L}$ THF was added to the pellet and the pellet was resuspended through gentle pipetting (at least 20 times). This suspension was then kept on a rotator for $20 \mathrm{~min}$. Finally, the particles were centrifuged $(3000 \mathrm{~g}, 30 \mathrm{~s})$, the supernatant was removed, and the process was repeated four times. After the final THF washing step, $200 \mu \mathrm{L}$ of high-purity water was used to resuspend the pellet through gentle pipetting.

For samples characterized by transmission electron microscopy (TEM) and atomic force microscopy (AFM), removal of the PS templates was achieved by overnight immersion in THF with constant agitation (on a rotator) during the third washing step, with the fourth THF washing step performed the next day. The samples were air-dried before analysis.

Molecular Dynamics (MD) Simulation Construction. A full description of the construction and preparation of the $\mathrm{Fe}^{\mathrm{III}}-\mathrm{TA}_{3}$ complexes can be found in our previous work. ${ }^{18}$ Briefly, the $\mathrm{Fe}^{\mathrm{III}}$ $\mathrm{TA}_{3}$ complexes were solvated in a cubic water box of $70 \times 70 \times 70 \AA^{3}$, with three sodium ions added to maintain net neutral charge. For the $2 \mathrm{M} \mathrm{NaCl}$ case study, 400 sodium and 400 chloride ions were added to the simulation box.

To study the effects of salt concentration on $\mathrm{Fe}^{\mathrm{III}}-\mathrm{TA}_{3}$ interaction, we modeled two complexes in proximity ( $\mathrm{Fe}^{\mathrm{III}}-\mathrm{TA}_{3}$ "dimers") in three starting configurations with an initial minimum heavy atom separation of $\sim 10 \AA$. These dimer systems were solvated in a cubic water box of $100 \times 100$ $\times 100 \AA^{3}$, with six sodium ions added to maintain net neutral charge. For the $2 \mathrm{M} \mathrm{NaCl}$ case study, 1200 sodium and 1200 chloride ions were added to the simulation box. 
MD Simulation Details. For all MD simulations, the published parameters from the Condensedphase optimized molecular potentials for atomistic simulation studies (COMPASS) force field ${ }^{19}$ were implemented in the open-source MD code LAMMPS. ${ }^{20}$ Missing angles and dihedrals for non-metal interactions were taken from the polymer consistent force field (PCFF). ${ }^{21}$ LennardJones parameters for iron were taken from the PCFF, and iron-TA bonded interactions were described by parameters developed in our previous work. ${ }^{18}$ Atomic partial charges on the iron and chelating oxygens were taken from quantum mechanics (QM) calculations, and the remaining atomic charges were calculated by the charge equilibration $(\mathrm{QEq})$ method. ${ }^{22}$ Lennard-Jones parameters for the sodium ion in the 9-6 functional form ${ }^{19}$ were taken from the INTERFACE force field, ${ }^{23}$ and for the chloride ion we applied a sigma value of $5.3 \AA$ and epsilon of $0.09 \mathrm{kcal} / \mathrm{mol}$, based on a comparison of radial distribution functions (RDFs), densities, and self-diffusion constants of $2 \mathrm{M} \mathrm{NaCl}$ solution simulations with experimental values. ${ }^{24,25}$ Electrostatic interactions beyond a $15.5-\AA$ cutoff were evaluated with the PPPM summation method with an accuracy of $10^{-5} \mathrm{kcal} / \mathrm{mol}$, and van der Waals interactions were assessed with an atom-based summation using a 15.5- $\AA$ cutoff and tail correction. MD simulations were run in the isothermal-isobaric ensemble $(N p T)$ using the Nosé-Hoover thermostat and barostat to maintain a temperature of $298 \mathrm{~K}$ and pressure of $1 \mathrm{~atm}$, with a time step of $1 \mathrm{fs}$ and an output frequency of $10 \mathrm{ps}$.

All simulations were run for up to $100 \mathrm{~ns}$, with at least five different initial velocity distributions for each solvent system studied. Equilibration was defined as the point at which the Fe ${ }^{\mathrm{III}}-\mathrm{TA}_{3}$ heavy-atom root mean square (RMS) deviation reached a steady value with a standard deviation of less than $1.5 \AA$. All analyses were performed on 10 ns equilibrated trajectories. Hydrogen bond statistics were calculated with a maximum donor-acceptor heavy atom distance of $3.5 \AA$ and a 
minimum angle of $120^{\circ}$. Self-diffusion coefficients were calculated from mean squared displacements (MSDs) in time-averaged $1 \mathrm{~ns}$ blocks taken over the entire trajectory using the Einstein relation. ${ }^{26}$

\section{RESULTS AND DISCUSSION}

$\mathrm{Fe}^{\mathrm{III}}$-TA capsules were prepared by depositing $\mathrm{Fe}^{\mathrm{III}}$-TA films on PS particle templates at different $\mathrm{NaCl}$ concentrations ranging from 0 to $2 \mathrm{M}$, and subsequent template removal by THF washing using an adapted experimental protocol from our previously reported methods. ${ }^{1}$ The surface morphology and thickness of the Fe $\mathrm{F}^{\mathrm{III}}$-TA capsules prepared under different ionic strengths were determined by AFM measurements (Figure 2a). When the salt concentrations were less than or equal to $0.75 \mathrm{M}$, the capsules featured a relatively smooth surface, similar to those prepared in the standard $0 \mathrm{M} \mathrm{NaCl}$ system. ${ }^{1}$ However, at salt concentrations greater than $0.75 \mathrm{M}$, the capsules became rougher and some grain-like microstructures were observed. This increased roughness likely results from the deposition of larger Fe $\mathrm{Fe}^{\mathrm{III}}$-TA complexes, and the extracted film thickness histograms clearly reveal the changes in film homogeneity (Figure $\mathbf{2 b}$ ). For example, when the salt concentration increased from 0 to $0.75 \mathrm{M}$, the RMS roughness remained smooth at $1.5 \mathrm{~nm}$. In contrast, the RMS roughness increased at each salt concentration studied above $0.75 \mathrm{M}$ (Figure S1). Moreover, the average film thickness increased across the $[\mathrm{NaCl}]$ range of 0 to $1 \mathrm{M}$ and increased minimally in the $[\mathrm{NaCl}]$ range of 1 to $2 \mathrm{M}$ (Figure 2c). Furthermore, the film thicknesses showed a bimodal distribution as the salt concentration increased. The first peak located at $\sim 11$ $\mathrm{nm}$ was ascribed to the homogenous and smooth portion of the Fe $\mathrm{e}^{\mathrm{III}}-\mathrm{TA}$ films as $11 \mathrm{~nm}$ is the median thickness value of the films prepared at lower salt concentrations. The second peak, which 
shifted to higher values at higher salt concentrations, was likely due to the embedding of larger $\mathrm{Fe}^{\mathrm{III}}-\mathrm{TA}$ complexes in the films, which resulted in increased film thickness and disruption of the film smoothness. Still, films thicker than $\sim 18 \mathrm{~nm}$ could not be formed simply by varying the ionic strength, likely because of shielding effects that prevent uniform and continuous deposition, similarly to what is seen for polyelectrolyte films. ${ }^{12}$

a
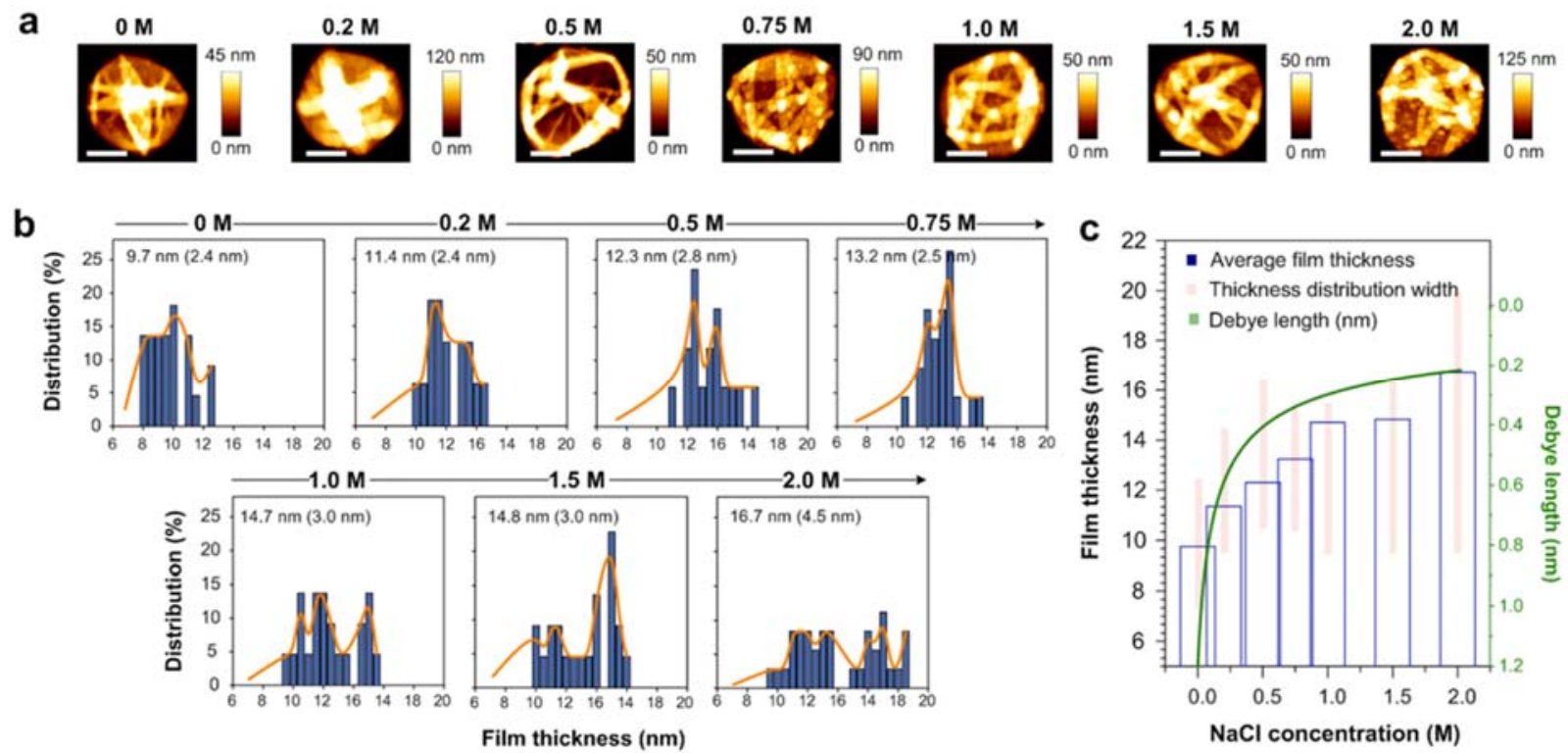

Figure 2. Influence of salt concentration on Fe $\mathrm{Fe}^{\mathrm{III}}$-TA film thickness. (a) Representative AFM images of the $\mathrm{Fe}^{\mathrm{III}}-\mathrm{TA}$ capsules prepared at different $\mathrm{NaCl}$ concentrations. (b) Film thickness distributions of $\mathrm{Fe}^{\mathrm{III}}$-TA capsules prepared at different $\mathrm{NaCl}$ concentrations, as determined by AFM. Thickness was characterized by the mean value and maximum distribution width given in parentheses. (c) Correlation between Fe ${ }^{\mathrm{III}}-\mathrm{TA}$ film thickness and Debye length (green line) at varying $\mathrm{NaCl}$ concentrations. The mean thickness is represented by the blue bar graph and distribution width of film thickness is represented by the error bars, $n=60$. 
The change in MPN film thickness and homogeneity with ionic strength is likely related to the screening of charges in solution, as seen for polyelectrolyte complexes. ${ }^{11}$ The screening of negative charges on TA, for example, may allow for closer packing of the ligands as they complex to $\mathrm{Fe}^{\mathrm{III}}$, ultimately leading to thicker films. The Debye length provides an indication of this change in screening. The inverse Debye length is calculated as follows: ${ }^{27}$

$k=\left(\frac{\sum_{i} \rho_{\infty i} e^{2} z_{i}^{2}}{\varepsilon_{0} \varepsilon k_{B} T}\right)^{1 / 2} m^{-1}$

where $\rho_{\infty i}$ is the concentration of ions in bulk solution, $e$ is the unit electronic charge, $z_{i}$ is the valance, $\varepsilon_{0}$ is the vacuum permittivity, $\varepsilon$ is the static permittivity, $k_{B}$ is the Boltzmann's constant, and $T$ is the absolute temperature. The change in the Fe ${ }^{\mathrm{III}}$-TA film thickness, as observed by AFM, showed qualitatively a similar growth profile to that observed for the Debye length as a function of salt concentration (Figure 2c), suggesting that the mechanism behind the increase in film thickness is closely related to the screening of charges by counterions.

a

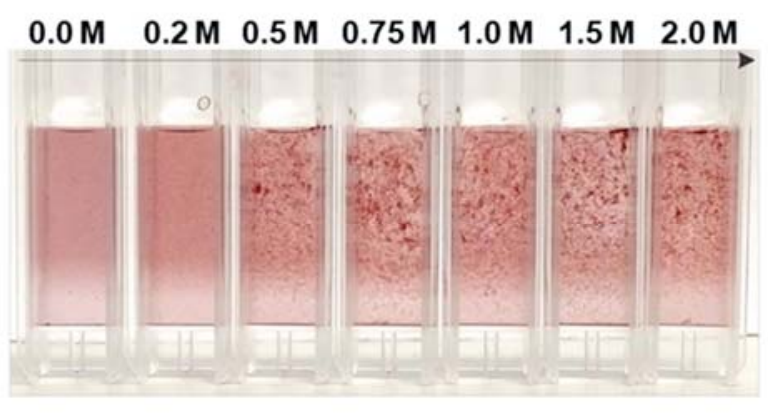

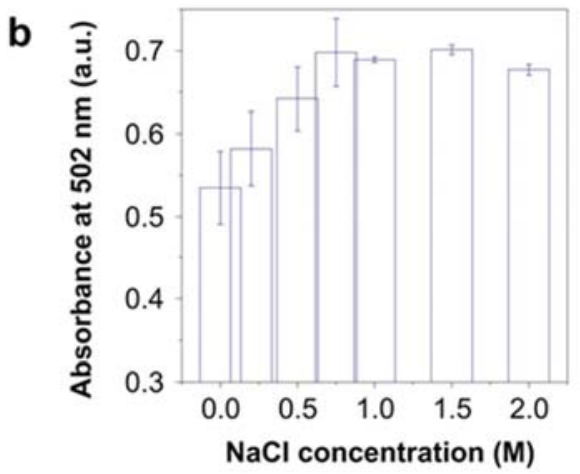

$\mathrm{NaCl}$ concentration (M)

Figure 3. Influence of salt concentration on the structure of the Fe $\mathrm{F}^{\mathrm{III}}-\mathrm{TA}$ complexes in solution. (a) Digital photos of the $\mathrm{Fe}^{\mathrm{III}}$-TA complexes at different $\mathrm{NaCl}$ concentrations, showing an increase 
in aggregate size with increasing $\mathrm{NaCl}$ concentrations (30 s after mixing of $\mathrm{FeCl}_{3}$ and $\mathrm{TA}$ ). (b) Absorbance of the $\mathrm{Fe}^{\mathrm{III}}$-TA complexes measured at $502 \mathrm{~nm}$ at different $\mathrm{NaCl}$ concentrations.

The similarity between the growth profile of the Fe $\mathrm{III}^{\mathrm{III}} \mathrm{TA}$ film thickness to the Debye length of the solutions suggest that the central mechanism of the film formation is related to the screening of charges in solution. Therefore, the properties of the Fe $\mathrm{F}^{\mathrm{III}}-\mathrm{TA}$ complexes in solution were investigated at different $\mathrm{NaCl}$ concentrations. The increase in $\mathrm{NaCl}$ concentration led to the formation of large aggregate clusters, which were visible to the naked eye (Figure 3a). Furthermore, the UV-vis absorbance profiles (measured at $502 \mathrm{~nm}$, which corresponds to the ligand-to-metal charge transfer (LMCT) for the tris-complex of Fe $\left.\mathrm{Fe}^{\mathrm{III}}-\mathrm{TA}\right)$ of the solutions suggest an increase in the interaction between complexes (Figure 3b). TEM confirmed the changes of the $\mathrm{Fe}^{\mathrm{III}}-\mathrm{TA}$ complexes prepared in different $\mathrm{NaCl}$ concentrations. When the $\mathrm{NaCl}$ concentration changed from 0 to $1 \mathrm{M}$, the individual $\mathrm{Fe}^{\mathrm{III}}$-TA nano-complexes agglomerated and formed clusters about $200 \mathrm{~nm}$ in diameter (Figure S2). At $2 \mathrm{M} \mathrm{NaCl}$, the Fe $\mathrm{Fe}^{\mathrm{III}}-\mathrm{TA}$ clusters agglomerated into a network of larger clusters $(>800 \mathrm{~nm})$. Similarly, small-angle X-ray scattering (SAXS) spectra of the $\mathrm{Fe}^{\mathrm{III}}$-TA complexes suggest an increase in network size with increasing $\mathrm{NaCl}$ concentrations along with an increase in the individual complex size (Figure 4). Specifically, the unit size of the complex shifted more towards the extended state, as seen in the increase in relative intensity at $0.103 \AA^{-1}$ (extended complex) when compared with the intensity at $0.110 \AA^{-1}$ (compact complex). These results collectively suggest that the Fe $\mathrm{F}^{\mathrm{III}}$-TA complexes undergo conformational changes as the $\mathrm{NaCl}$ concentration increases, which allows for bridging between complexes in solution. 

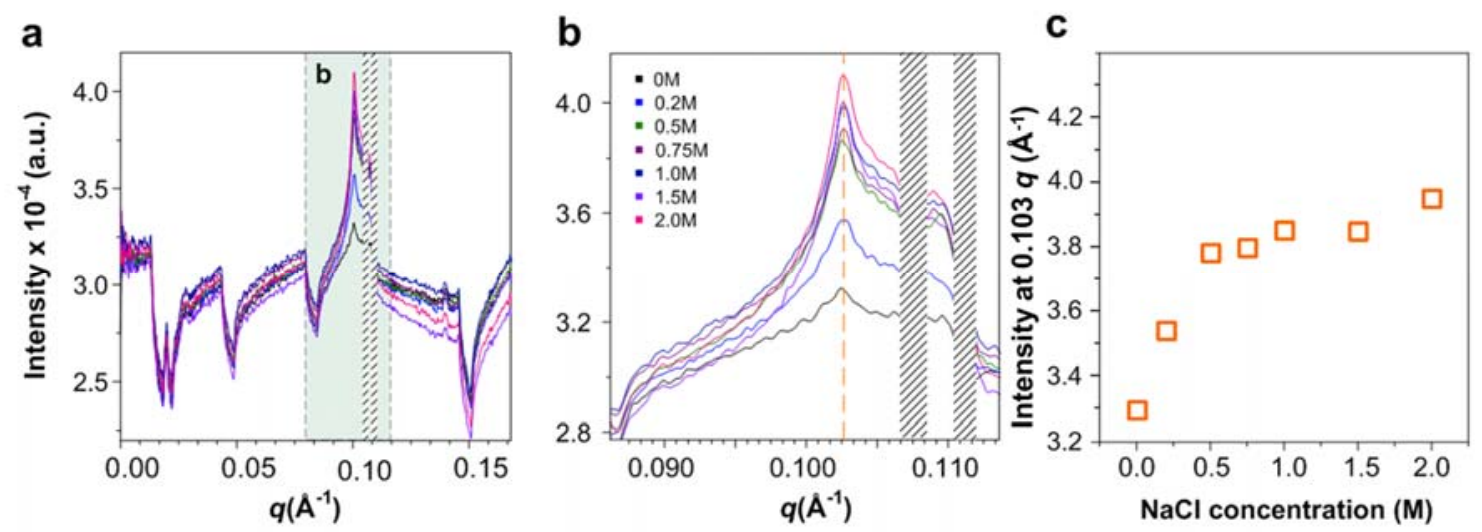

Figure 4. The influence of salt concentration on the structure of $\mathrm{Fe}^{\mathrm{III}}-\mathrm{TA}$ complexes in solution. (a) and (b) SAXS spectra of the Fe $\mathrm{F}^{\mathrm{III}}$-TA complexes in different $\mathrm{NaCl}$ solutions. The detector gaps are represented by the grey shaded areas. The intensity $\sim 0.08-.12 \AA^{-1}$ increased with increasing concentrations of $\mathrm{NaCl}$, and specifically the relative intensity of the peak at $0.103 \AA^{-1}$ (extended state) increased in relation to the intensity of the peak at $0.110 \AA^{-1}$ (compact state). (c) Intensity of the peak at $0.103 \AA^{-1}$ at varying salt concentrations (same data as b represented in a different form).

Optical waveguide light-mode spectroscopy (OWLS) measurements revealed the mass of the films as a function of ionic strength. The basic principle of OWLS relies on measuring the mode spectrum of an optical waveguide, the surface of which is sensitive to changes in the refractive index of the coating solution, and the change in refractive index can be used to calculate the change in adsorbed mass. ${ }^{28}$ In the present study, Fe ${ }^{\mathrm{III}}$-TA solutions were immediately injected into the OWLS instrument at a flow rate of $20 \mu \mathrm{L} \mathrm{min}^{-1}$ after mixing the $\mathrm{Fe}^{3+}$ ions and TA together in the $\mathrm{NaCl}$ solution at the desired concentration. A change in adsorbed mass was observed once the 
complexes reached the sensor chip and formed the MPN film. Excess complexes were washed off under the same flow rate using the respective $\mathrm{NaCl}$ solutions, where an interesting difference in the amount of excess MPN complex (that is not part of the deposited film) was observed among the $0.1 \mathrm{M}, 0.5 \mathrm{M}$, and $1.0 \mathrm{M}$ systems. This difference is likely related to a cross-over in deposition mechanism between low and high ionic strengths. The mass of the Fe $\mathrm{F}^{\mathrm{III}}-\mathrm{TA}$ in the films increased with increasing salt concentration (Figure 5), which is consistent with the AFM results (Figure 2). AFM analysis was also performed on the OWLS silica substrate after film formation for the sample prepared in $1 \mathrm{M} \mathrm{NaCl}$ to determine if excess complexes were interfering with the measurements. The AFM analysis revealed a smooth surface with few excess complexes, thus confirming that film formation proceeded in a similar way to the MPN films formed outside of the OWLS instrument (Figure 5b inset). Note that the corrugations in the AFM image are part of the optical waveguide layer.
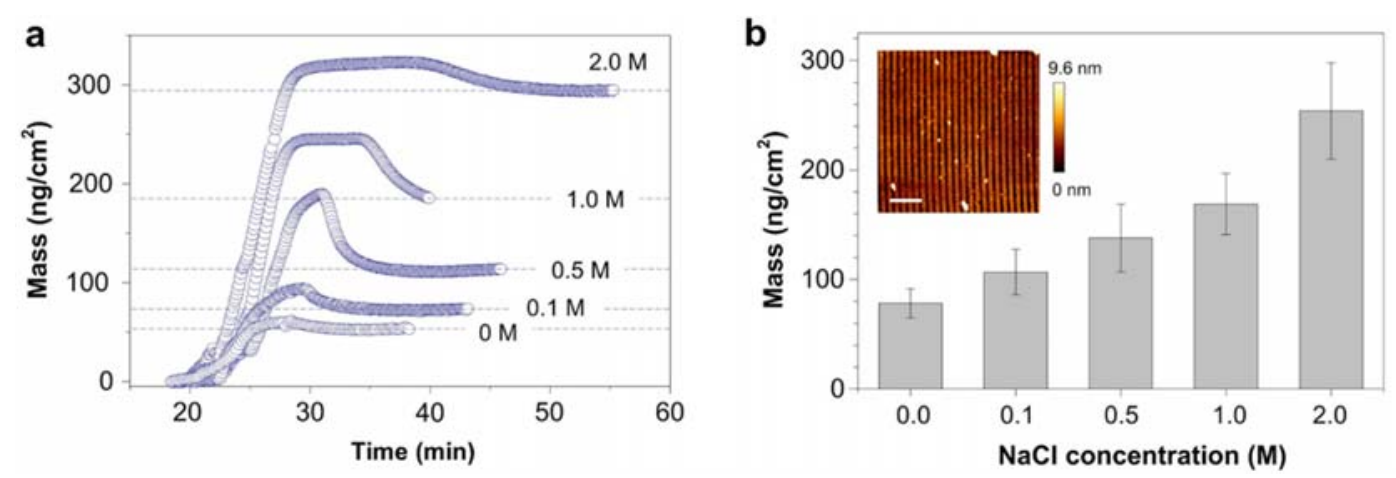

Figure 5. Influence of salt concentration on the mass of Fe $\mathrm{III}_{-\mathrm{TA}}$ deposited as a film. (a) Mass change of the $\mathrm{Fe}^{\mathrm{III}}$-TA films over time in different $\mathrm{NaCl}$ concentrations and (b) final mass values of the films on the OWLS substrates. The inset image in (b) shows the surface of the MPN-coated OWLS substrate. Scale bar is $2 \mu \mathrm{m}$. 
MD simulations were used to elucidate the configuration of monomer and dimer Fe $\mathrm{e}^{\mathrm{III}}-\mathrm{TA}$ complexes in $0 \mathrm{M}$ (Figure 6a and b) and $2 \mathrm{M} \mathrm{NaCl}$ (Figure 6c and d), respectively. Calculations of the $\mathrm{Fe}^{\mathrm{III}}-\mathrm{TA} 3$ self-diffusion constants revealed that the $\mathrm{Fe}^{\mathrm{III}}-\mathrm{TA}_{3}$ complex diffused $43 \%$ more slowly in $2 \mathrm{M} \mathrm{NaCl}$ solution than in pure water (Figure S3). Minor differences in the solventaccessible surface area (SASA), radius of gyration $\left(R_{\mathrm{g}}\right)$, intramolecular hydrogen bonds, and intramolecular aromatic ring contacts were observed between the pure water and the $2 \mathrm{M} \mathrm{NaCl}$ systems (Table S1). However, the root mean square fluctuation (RMSF) of heavy atoms was 17\% smaller in the $2 \mathrm{M} \mathrm{NaCl}$ system than in the pure water system. Oxygen-oxygen intramolecular RDFs revealed that the $\mathrm{Fe}^{\mathrm{III}}-\mathrm{TA}_{3}$ was more structured in $2 \mathrm{M} \mathrm{NaCl}$, and both the water-Fe $\mathrm{F}_{-}^{\mathrm{III}}-\mathrm{TA}_{3}$ oxygen-oxygen RDFs and the water-Fe $\mathrm{F}^{\mathrm{III}}-\mathrm{TA}_{3}$ hydrogen bond occupancies demonstrated that there were fewer interactions between $\mathrm{Fe}^{\mathrm{III}}-\mathrm{TA}_{3}$ and water in $2 \mathrm{M} \mathrm{NaCl}$. Similar to the monomer systems, the RDFs of the $\mathrm{Fe}^{\mathrm{III}}-\mathrm{TA} 3$ dimer systems showed more structuring, and the heavy-atom RMSF was smaller in the $2 \mathrm{M} \mathrm{NaCl}$ system than that in the pure water system (Table S2). However, the $R_{\mathrm{g}}$, end-to-end distance, and SASA were larger in the $2 \mathrm{M} \mathrm{NaCl}$ system than those in the pure water system, and there was a slight decrease in the dimer contact area and number of intermolecular aromatic ring contacts. The free energy plots of $R_{\mathrm{g}}$ and end-to-end distance revealed that there is a distinct population of structures of larger $R_{\mathrm{g}}$ and longer end-to-end distance in the 2 $\mathrm{M} \mathrm{NaCl}$ system. In other words, in $2 \mathrm{M} \mathrm{NaCl}$, the galloyl groups extend away from the bulk of the $\mathrm{Fe}^{\mathrm{III}}-\mathrm{TA}_{3}$ dimer, whereas in $0 \mathrm{M} \mathrm{NaCl}$, the galloyl groups of the $\mathrm{Fe}^{\mathrm{III}}-\mathrm{TA}_{3}$ dimer are directed toward the Fe center, resulting in a packed configuration (Figure 6). These results indicate that the presence of $\mathrm{NaCl}$ changes the configuration of the $\mathrm{Fe}^{\mathrm{III}}-\mathrm{TA}$ complexes from packed smaller structures to extended larger structures, which we infer leads to greater interaction between multiple complexes. Moreover, in $2 \mathrm{M} \mathrm{NaCl}$, each $\mathrm{Fe}^{\mathrm{III}}-\mathrm{TA}_{3}$ is surrounded by a layer of sodium 
ions that associate with the oxygens chelating the $\mathrm{Fe}^{\mathrm{III}}$ center, and with the hydroxyl oxygens of the galloyl groups (Figure 6e and f).
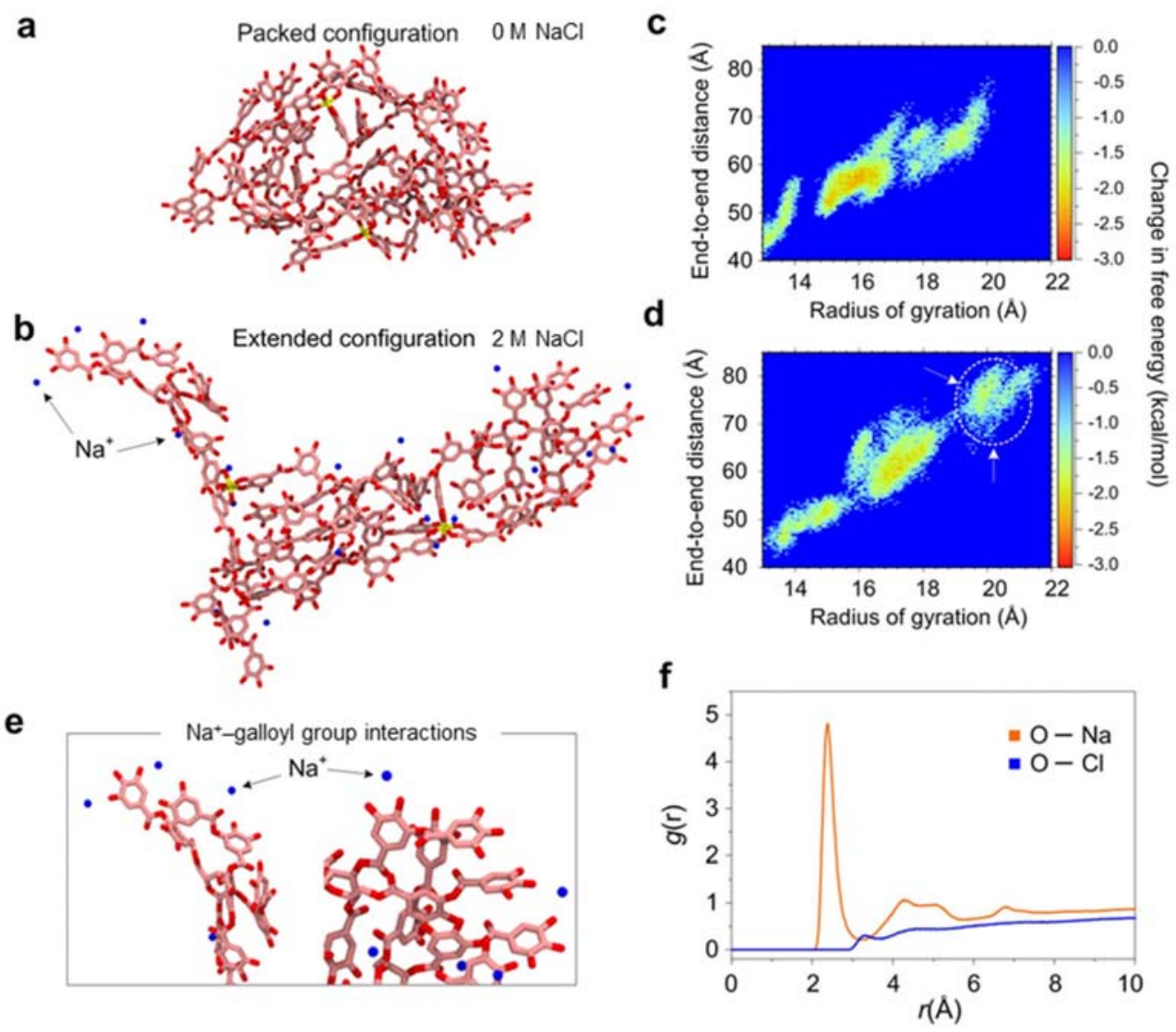

Figure 6. Molecular interactions of the $\mathrm{Fe}^{\mathrm{III}}-\mathrm{TA}_{3}$ dimer systems at the atomic level. Typical equilibrium configurations of the $\mathrm{Fe}^{\mathrm{III}}-\mathrm{TA}_{3}$ dimer in (a) $0 \mathrm{M}$ and (b) $2 \mathrm{M} \mathrm{NaCl}$, and the corresponding changes in free energy as a function of end-to-end distance and radius of gyration for the $\mathrm{Fe}^{\mathrm{III}}-\mathrm{TA}_{3}$ dimer for $0 \mathrm{M}$ (c) and $2 \mathrm{M}$ (d) $\mathrm{NaCl}$. (e) Representative $\mathrm{Na}^{+}$-galloyl interactions $\left(\mathrm{Na}^{+}\right.$ions in the first hydration shell are shown as blue spheres). For clarity, water, chloride ions, and hydrogen atoms are not shown. (f) RDF of the $\mathrm{Fe}^{\mathrm{III}}-\mathrm{TA}_{3}$ complex dimer hydroxyl oxygens to $\mathrm{Na}^{+}$and $\mathrm{Cl}^{-}$ions in $2 \mathrm{M} \mathrm{NaCl}$. 
The MD simulations demonstrate that the large number of sodium ions, when compared with chloride ions, in the first hydration shell creates a positively charged layer around the tannic acid that may prevent aggregation of the galloyl groups (Table S2). This ion shell around the Fe ${ }^{\mathrm{III}}-\mathrm{TA} 3$ complex likely disrupts hydrogen bonding between the $\mathrm{Fe}^{\mathrm{III}}-\mathrm{TA}_{3}$ complex monomers, leading to free hydroxyl reaction sites that can coordinate with other free $\mathrm{Fe}^{\mathrm{III}}$ ions and/or complexes when compared to the case observed for the $\mathrm{Fe}^{\mathrm{III}}-\mathrm{TA}_{3}$ complex in $0 \mathrm{M} \mathrm{NaCl}$ water. The extension of galloyl groups outward toward the bulk solution suggests that the tannic acid ligands have a higher solubility at higher ionic strengths. This could be due to a change in hydrogen bonding in water owing to the presence of ions, which for the case of tannic acid allows galloyl groups to become more exposed in solution and provide an anchoring point for further complexation. We suggest it is this mechanism that results in the experimentally observed thicker and rougher MPN films in 2 $\mathrm{M} \mathrm{NaCl}$. Additionally, the packed conformation of the $\mathrm{Fe}^{\mathrm{III}}-\mathrm{TA}_{3}$ complex observed in pure water likely allowed for the formation of smooth MPN films under these conditions.

\section{CONCLUSIONS}

The present work examined the effects of ionic strength on Fe $\mathrm{F}^{\mathrm{III}}-\mathrm{TA}$ complexes in solution and on the deposition of MPN films. The film characteristics were studied through the formation of hollow capsules. Increasing the $\mathrm{NaCl}$ concentration to up to $1 \mathrm{M}$ led to thicker films and further increases above $1 \mathrm{M}$ led to rougher films. SAXS and MD simulations of monomer and dimer Fe $\mathrm{F}^{\mathrm{III}}$ $\mathrm{TA}_{3}$ complexes in 0 and $2 \mathrm{M} \mathrm{NaCl}$ provided information on the structural differences in the chelation complexes resulting from electrostatic screening induced by ions. At higher ionic strengths, the galloyl groups of $\mathrm{Fe}^{\mathrm{III}}-\mathrm{TA}_{3}$ complexes could extend further into the bulk solution 
away from the metal center. This was inferred to lead to subsequent chelation with other Fe ${ }^{\mathrm{III}}$ ions and/or complexes, ultimately leading to thicker and rougher films. In contrast, at lower ionic strengths $(0 \mathrm{M} \mathrm{NaCl})$, the complexes exhibited a packed conformation, thereby limiting film growth and allowing for the formation of smooth films. For example, at $\mathrm{NaCl}$ concentrations of less than $1 \mathrm{M}$, the films were smooth; the RMS roughness was nearly identical to the radius of gyration of tannic acid $(1.5 \mathrm{~nm}) .{ }^{1}$ Contrarily, at higher ionic strengths, above $1 \mathrm{M} \mathrm{NaCl}$, the extended conformation of tannic acid yielded rougher films. This study focused on Fe ${ }^{\mathrm{III}}-\mathrm{TA}$ complexes, however, it is expected that other metals will behave in a similar way to the studied systems. Our results provide new insights into the behavior of $\mathrm{Fe}^{\mathrm{III}}-\mathrm{TA}_{3}$ complexes in solution and the influence of ionic strength on the deposition of MPN films. Finally, metal-phenolic interactions have applications outside of thin films such as seeding the growth of metal-organic frameworks around various nanoparticles ${ }^{29}$ and generating responsive hydrogels. ${ }^{30}$ Therefore, we believe that this work may contribute to diverse fields, ranging from metal-organic frameworks to thin films, to hydrogels.

\section{ASSOCIATED CONTENT}

Supporting Information. Additional experimental details and additional experimental data, tables of MD simulation results.

\section{AUTHOR INFORMATION}

\section{Corresponding Author}

*E-mail: fcaruso@unimelb.edu.au. 


\section{Author Contributions}

J.G. and J.J.R. contributed equally to this work. The manuscript was written through contributions of all authors. All authors have given approval to the final version of the manuscript.

\section{ACKNOWLEDGMENT}

This research was conducted and funded by the Australian Research Council (ARC) Centre of Excellence in Convergent Bio-Nano Science and Technology (project number CE140100036). This work was also supported by the ARC under the Australian Laureate Fellowship (F.C., FL120100030) and Discovery Project (F.C., DP130101846) schemes. This work was performed in part at the Materials Characterisation and Fabrication Platform (MCFP) at The University of Melbourne and the Victorian Node of the Australian National Fabrication Facility (ANFF). We acknowledge F. Tian, Q. Dai, D. Song, Y. Ju, M. Penna, and P. Charchar for helpful discussions. A.J.C. and I.Y. acknowledge the generous allocation of high-performance computational resources from the Australian National Computational Infrastructure (NCI), the Pawsey Supercomputing Centre, and the Victorian Life Sciences Computational Initiative (VLSCI). Part of this work was performed at the SAXS beamline of the Australian Synchrotron.

\section{REFERENCES}

(1) Ejima, H.; Richardson, J. J.; Liang, K.; Best, J. P.; van Koeverden, M. P.; Such, G. K.; Cui, J.; Caruso, F. One-Step Assembly of Coordination Complexes for Versatile Film and Particle Engineering. Science 2013, 341, 154-157. 
(2) Rahim, M. A.; Kempe, K.; Müllner, M.; Ejima, H.; Ju, Y.; van Koeverden, M. P.; Suma, T.; Braunger, J. A.; Leeming, M. G.; Abrahams, B. F.; Caruso, F. Surface-Confined Amorphous Films from Metal-Coordinated Simple Phenolic Ligands. Chem. Mater. 2015, 27, 5825-5832.

(3) Guo, J.; Ping, Y.; Ejima, H.; Alt, K.; Meissner, M.; Richardson, J. J.; Yan, Y.; Peter, K.; von Elverfeldt, D.; Hagemeyer, C. E.; Caruso, F. Engineering Multifunctional Capsules through the Assembly of Metal-Phenolic Networks. Angew. Chem., Int. Ed. 2014, 53, 5546-5551.

(4) Ejima, H.; Richardson, J. J.; Caruso, F. Metal-Phenolic Networks as a Versatile Platform to Engineer Nanomaterials and Biointerfaces. Nano Today 2016, 12, 136-148.

(5) Park, C.; Yang, B. J.; Jeong, K. B.; Kim, C. B.; Lee, S.; Ku, B. C. Signal-Induced Release of Guests from a Photolatent Metal-Phenolic Supramolecular Cage and Its Hybrid Assemblies. Angew. Chem., Int. Ed. 2017, 56, 5485-5489.

(6) Sunda, W.; Huntsman, S. Effect of pH, Light, and Temperature on Fe-EDTA Chelation and Fe Hydrolysis in Seawater. Mar. Chem. 2003, 84, 35-47.

(7) Harrison, S. M.; Bers, D. M. The Effect of Temperature and Ionic Strength on the Apparent Ca-Affinity of EGTA and the Analogous Ca-Chelators BAPTA and Dibromo-BAPTA. Biochim. Biophys. Acta 1987, 925, 133-143.

(8) Zeng, H.; Hwang, D. S.; Israelachvili, J. N.; Waite, J. H. Strong Reversible Fe ${ }^{3+}$-Mediated Bridging between Dopa-Containing Protein Films in Water. Proc. Natl. Acad. Sci. U. S. A. 2010, $107,12850-12853$. 
(9) Mettu, S.; Zhou, M.; Tardy, B. L.; Ashokkumar, M.; Dagastine, R. R. Temperature Dependent Mechanical Properties of Sir, Oil and Water Filled Microcapsules Studied by Atomic Force Microscopy. Polymer 2016, 102, 333-341.

(10) Lu, L.; Li, Y.; Lu, X. Kinetic Study of the Complexation of Gallic Acid with Fe (II). Spectrochim. Acta, Part A 2009, 74, 829-834.

(11) McAloney, R. A.; Sinyor, M.; Dudnik, V.; Goh, M. C. Atomic Force Microscopy Studies of Salt Effects on Polyelectrolyte Multilayer Film Morphology. Langmuir 2001, 17, 6655-6663.

(12) Kovacevic, D.; Van der Burgh, S.; De Keizer, A.; Cohen Stuart, M. Kinetics of Formation and Dissolution of Weak Polyelectrolyte Multilayers: Role of Salt and Free Polyions. Langmuir 2002, 18, 5607-5612.

(13) Shamoun, R. F.; Reisch, A.; Schlenoff, J. B. Extruded Saloplastic Polyelectrolyte Complexes. Adv. Funct. Mater. 2012, 22, 1923-1931.

(14) Wang, Q.; Schlenoff, J. B. The Polyelectrolyte Complex/Coacervate Continuum. Macromolecules 2014, 47, 3108-3116.

(15) Salomäki, M.; Kankare, J. Specific Anion Effect in Swelling of Polyelectrolyte Multilayers. Macromolecules 2008, 41, 4423-4428.

(16) Salomäki, M.; Laiho, T.; Kankare, J. Counteranion-Controlled Properties of Polyelectrolyte Multilayers. Macromolecules 2004, 37, 9585-9590.

(17) Xiao, F.; Pagliaro, M.; Xu, Y.; Liu, B. Layer-by-Layer Assembly of Versatile Nanoarchitectures with Diverse Dimensionality: A New Perspective for Rational Construction of Multilayer Assemblies. Chem. Soc. Rev. 2016, 45, 3088-3121. 
(18) Guo, J.; Tardy, B. L.; Christofferson, A. J.; Dai, Y.; Richardson, J. J.; Zhu, W.; Hu, M.; Ju, Y.; Cui, J.; Dagastine, R. R.: Yarovsky, I.; Caruso, F. Modular Assembly of Superstructures from Polyphenol-Functionalized Building Blocks. Nat. Nanotechnol. 2016, 11, 1105-1111.

(19) Sun, H. COMPASS: An ab Initio Force-Field Optimized for Condensed-Phase Applications_Overview with Details on Alkane and Benzene Compounds. J. Phys. Chem. B 1998, $102,7338-7364$.

(20) Plimpton, S. Fast Parallel Algorithms for Short-Range Molecular Dynamics. J. Comput. Phys. 1995, 117, 1-19.

(21) Sun, H.; Mumby, S. J.; Maple, J. R.; Hagler, A. T. An ab Initio CFF93 All-Atom Force Field for Polycarbonates. J. Am. Chem. Soc. 1994, 116, 2978-2987.

(22) Rappe, A. K.; Goddard, W. A. Charge Equilibration for Molecular Dynamics Simulations. J. Phys. Chem. 1991, 95, 3358-3363.

(23) Heinz, H.; Lin, T.-J.; Kishore Mishra, R.; Emami, F. S. Thermodynamically Consistent Force Fields for the Assembly of Inorganic, Organic, and Biological Nanostructures: The INTERFACE Force Field. Langmuir 2012, 29, 1754-1765.

(24) Ignaczak, A.; Gomes, J. A. N. F.; Cordeiro, M. N. D. S. Quantum and Simulation Studies of X-( $\left.\mathrm{H}_{2} \mathrm{O}\right)$ n Systems. Electrochim. Acta 1999, 45, 659-673.

(25) Lide, D. R. Handbook of Chemistry and Physics, Internet Version, 87th ed; Taylor and Francis: Boca Raton, FL, 2007.

(26) Allen, M. P.; Tildesley, D. J. Computer Simulation of Liquids; Oxford University Press: New York, 1980. 
(27) Israelachvili, J. N. Intermolecular and Surface Forces; Academic Press: 2011.

(28) Kurrat, R.; Textor, M.; Ramsden, J.; Böni, P.; Spencer, N. Instrumental Improvements in Optical Waveguide Light Mode Spectroscopy for the Study of Biomolecule Adsorption. Rev. Sci. Instrum. 1997, 68, 2172-2176.

(29) Zhou, J.; Wang, P.; Wang, C.; Goh, Y. T.; Fang, Z.; Messersmith, P. B.; Duan, H. Versatile Core-Shell Nanoparticle@Metal-Organic Framework Nanohybrids: Exploiting Mussel-Inspired Polydopamine for Tailored Structural Integration. ACS Nano 2015, 9, 6951-6960.

(30) Holten-Andersen, N.; Harrington, M. J.; Birkedal, H.; Lee, B. P.; Messersmith, P. B.; Lee, K. Y. C.; Waite, J. H. pH-Induced Metal-Ligand Cross-Links Inspired by Mussel Yield SelfHealing Polymer Networks with Near-Covalent Elastic Moduli. Proc. Natl. Acad. Sci. U. S. A. 2011, 108, 2651-2655. 
TOC:

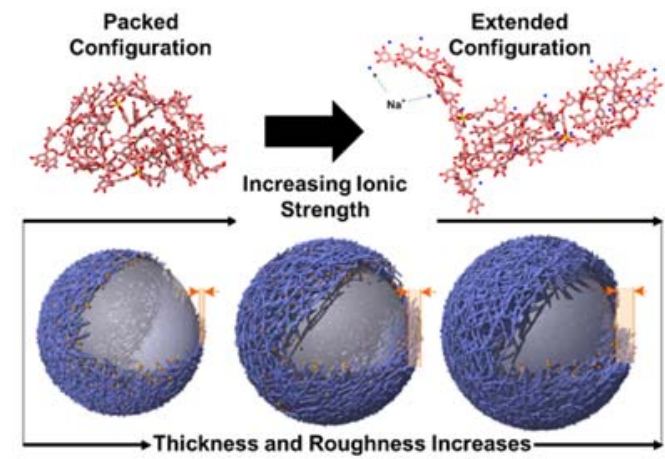

